\title{
On the Arrow-Hahn utility representation method
}

\author{
Michele Gori*and Giulio Piangiani ${ }^{\dagger}$
}

September 17, 2009

\begin{abstract}
In this paper we characterize metric spaces used in Beardon's generalization of ArrowHahn utility representation method as generalized Peano continua. For continuous preference relations defined on such metric spaces, we further construct an upper semi-continuous utility function which explicitly depends on the distance.
\end{abstract}

Keywords: preference relation; utility function; convex metric space; generalized Peano continuum.

JEL Classification Numbers: D11.

2000 Mathematics Subject Classification: 54D05.

\section{Introduction}

A binary relation $\succeq$ defined on a set $X$ is called a preference relation on $X$ if it is transitive and complete. Given a preference relation $\succeq$ on $X$ and $x_{1}, x_{2} \in X$, we write $x_{2} \succ x_{1}$, if $x_{2} \succeq x_{1}$ and $x_{1} \nsucceq x_{2}$, while we write $x_{2} \sim x_{1}$, if $x_{2} \succeq x_{1}$ and $x_{1} \succeq x_{2}$. A function $u: X \rightarrow \mathbb{R}$ is called utility function representing $\succeq$ if, for every $x_{1}, x_{2} \in X, x_{2} \succeq x_{1}$ if and only if $u\left(x_{2}\right) \geq u\left(x_{1}\right)$.

When $X$ is endowed with a topology $\tau$, an interesting and widely studied problem is to find conditions on $\tau$ and $\succeq$ which imply the existence of continuous utility functions. Of course a necessary condition is that $\succeq$ is continuous, that is, for every $x \in X$,

$$
C^{+}(x)=\{y \in X: y \succeq x\} \quad \text { is closed, }
$$

and

$$
O^{+}(x)=\{y \in X: y \succ x\} \quad \text { is open. }
$$

Given a continuous preference relation $\succeq$ on a topological space $(X, \tau)$, a continuous representation of it can be found if either $(X, \tau)$ is second countable (see [7], Proposition 3) or $(X, \tau)$ is separable and connected (see [8], Statement 6, p. 43) or $(X, \tau)$ is separable and locally connected (see [6], Theorem 1$)$ or $(X, \tau)$ is path-connected and $\succeq$ is countably bounded (see [13], Theorem 3). We point out that all the above quoted results concern the existence of continuous utility functions but do not provide any effective way of constructing them.

\footnotetext{
*Dipartimento di Matematica per le Decisioni, Università di Firenze, e-mail: michele.gori@unifi.it.

${ }^{\dagger}$ Dipartimento di Matematica per le Decisioni, Università di Firenze, e-mail: giulio.pianigiani@unifi.it.
} 
On the contrary, the so-called Euclidean distance approach allows the construction of a utility function from the preference relation in a simpler and more explicit way (see [1] for detailed information and further references on the topic). This method was first introduced by Arrow and Hahn (see [2], pp. 82-87) to find a lower semi-continuous (but not necessarily continuous) utility function for continuous preference relations defined on convex and closed subsets of a Euclidean space. The upper semi-continuity can be obtained by assuming that the preference relation is locally non-satiated.

Beardon in [3] shows that such a method continues to work in a more general setting in which, as the author emphasizes, "no assumption about convexity, linear spaces, connectedness is made" (see [3], p. 370). In fact, he proves that Arrow and Hahn's approach provides a lower semi-continuous (but not necessarily continuous) utility function whenever the preference relation is continuous and defined on a metric space whose metric satisfies the following two properties

$$
\text { every closed ball is compact, }{ }^{1}
$$

if $x_{1}, x_{2} \in X$ with $x_{1} \neq x_{2}$, then each neighbourhood of $x_{2}$ contains a point closer to $x_{1}$ than $x_{2}$.

Moreover, in this setting too, local non-satiation implies upper semi-continuity of the utility function.

The proof of this result follows the main steps of Arrow and Hahn's proof. First it is proved that the restriction of the preference relation to any closed upper contour set $C^{+}(x)$ can be represented by a utility function defined via a simple formula involving the distance (see [3], Lemma 3). Then, by a suitable extension procedure, a utility function on the whole space is obtained by the utility functions previously constructed (see [3], Theorem 1).

The description of the Euclidean distance approach mentioned above surely suggests some issues. First of all, as pointed out by Bridges and Mehta, "bearing in mind that we expect a consumer to seek consumption bundles that maximize the value of his utility function (subject to budgetary constraints), we would prefer to construct a utility function that is upper, rather than lower, semi-continuous" (see [5], pp. 28-29). Moreover, as underlined by Alcantud and Mehta in [1], the extension procedure used in [2] and [3] vitiates the distance approach because it does not allow to have a utility function directly defined in terms of distance. Finally the properties of metric spaces whose distance satisfies (1) and (2) should be carefully analyzed.

Moving from the above considerations, in the first part of the paper we provide a characterization of the metric spaces considered by Beardon and we show that (1) and (2) imply several topological properties. In fact in Theorem 1 we prove that a topological space $(X, \tau)$ admits a distance which induces $\tau$ and satisfies (1) and (2) if and only if it is a generalized Peano continuum, that is, a locally compact, locally connected, connected and metrizable topological space. Furthermore we show that such topological spaces always admit a convex metric which satisfies (1) and (2) and preserves the original topology. These facts stress, in particular, that connectedness of the space is implicitly used in Beardon's representation theorem and suggest that such a topological property has an important role in the construction of the utility function through the distance.

In the second part of the paper, we consider continuous preference relations defined on a metric space satisfying (1) and (2) and we propose a simple formula in terms of distance that

\footnotetext{
${ }^{1}$ Sometimes metric spaces whose closed balls are compact are called proper metric spaces.
} 
defines an upper semi-continuous (but not necessarily continuous) utility function. Furthermore we find conditions on the preference relations in order to obtain continuity, as well.

We note that, within the Euclidean distance approach, Bridges and Mehta propose a way to construct an upper semi-continuous representation of a continuous preference relation defined on a closed and convex subset of a Euclidean space (see [5], Theorem 2.1.6). However the utility function they construct is obtained by an extension procedure that makes that function not directly depend on the distance. Finally Alcantud and Mehta in [1] consider continuous preferences on a proper open convex subset $X$ of a Banach space. In that framework they prove that, under technical assumptions, the function that assigns to each element $x$ of $X$ the distance of $C^{+}(x)$ from a fixed element in the complement of $X$ is continuous and represents the given preference (see [1], Theorems 1, 2, 3). It is an open problem to understand the relation between the methods developed by Alcantud and Mehta and the results of the present paper.

\section{Topological results}

Let $(X, d)$ be a metric space. In what follows by $B(x, r)$ and $B[x, r]$ we mean the open ball and the closed ball of center $x \in X$ and radius $r>0$, that is,

$$
B(x, r)=\{y \in X: d(x, y)<r\}, \quad B[x, r]=\{y \in X: d(x, y) \leq r\} .
$$

For $A \subseteq X$, we denote by $\bar{A}$ the closure of $A$. For every nonempty set $A \subseteq X$ and $x \in X$ by $d(x, A)$ we denote the distance between $x$ and the set $A$, that is

$$
d(x, A)=\inf \{d(x, y): y \in A\} .
$$

Note that $d(x, A)=d(x, \bar{A})$ and that $A \subseteq B$ implies $d(x, A) \geq d(x, B)$. Moreover if $A$ is compact then the distance is really obtained, that is, there exists $a \in A$ such that $d(x, A)=$ $d(x, a)$.

A Peano continuum is a compact, locally connected, connected and metrizable topological space. A generalized Peano continuum is a locally compact, locally connected, connected and metrizable topological space. A metric $d$ on $X$ is called convex if, for every $x, y \in X, x \neq y$, there exists $z \in X$ such that

$$
d(x, z)+d(y, z)=d(x, y) \text { and } \quad x \neq z \neq y,
$$

while is called segmented convex if, for every $x, y \in X, x \neq y$, there exists $\left[t_{1}, t_{2}\right] \subseteq \mathbb{R}$ and an isometry $\gamma:\left[t_{1}, t_{2}\right] \rightarrow X$ such that $\gamma\left(t_{1}\right)=x$ and $\gamma\left(t_{2}\right)=y$. A metric $d$ is complete if the metric space $(X, d)$ is complete. Any segmented convex metric is convex and any complete convex metric is segmented convex (see [11]).

Menger [11] has raised the question whether for every Peano continuum it is possible to define a convex metric preserving the original topology. The affirmative answer to this question was given by Bing [4] and Moise [12]. Later on, Tominaga and Tanaka [15] gave an affirmative answer to Menger's question for generalized Peano continua as well.

The following theorem shows that assumptions (1) and (2) on $d$ are equivalent to assume that the metric space $(X, d)$ is a generalized Peano continuum.

Theorem 1. Let $(X, \tau)$ be a topological space. Then the three following conditions are equivalent: 
a. $(X, \tau)$ is a generalized Peano continuum,

b. there exists a complete convex metric d on X satisfying (1) and (2) which induces $\tau$,

c. there exists a metric $d$ on $X$ satisfying (1) and (2) which induces $\tau$.

Proof. $\mathbf{a} \Rightarrow \mathbf{b}$. The existence of a complete convex metric $d$ on $X$ that preserves its topology is given in [15]. The validity of (1) is given in [9]. The validity of (2) follows from the fact that a complete convex metric is segmented convex.

$\mathbf{b} \Rightarrow \mathbf{c}$. Straightforward.

$\mathbf{c} \Rightarrow \mathbf{a}$. Let $d$ be a metric which induces the topology $\tau$ and satisfies (1) and (2). Condition (1) implies that $(X, d)$ is locally compact. We claim that any closed ball in $(X, d)$ is connected. Suppose, by contradiction, that there exist $x \in X$ and $r>0$ such that $B[x, r]$ is not connected. Then there exist $Y, Z$ open subsets of $B[x, r]$ such that $Y, Z \neq \varnothing, Y \cap Z=\varnothing$ and $Y \cup Z=$ $B[x, r]$. Of course $Y, Z$ are closed subsets of $B[x, r]$ too. Then we can find $Y^{o p}, Z^{o p}$ open subsets of $X$ and $Y^{c l}, Z^{c l}$ closed subsets of $X$ such that

$$
Y=Y^{o p} \cap B[x, r]=Y^{c l} \cap B[x, r], \quad Z=Z^{o p} \cap B[x, r]=Z^{c l} \cap B[x, r] .
$$

Without loss of generality, we can assume $x \in Y$ and then, since $Y \cap Z=\varnothing$, if $z \in Z$ then $z \neq x$. Note that, for every $z \in Z$, there exists $\widetilde{z} \in Z$ such that $d(x, \widetilde{z})<d(x, z)$. In fact if $z \in Z$, then $d(x, z) \leq r$ and $z \in Z^{o p}$. By (2) there exists $\widetilde{z} \in Z^{o p}$ such that $d(x, \widetilde{z})<d(x, z) \leq r$. In particular $\widetilde{z} \in Z$.

From (1) we have that $Z=Z^{c l} \cap B[x, r]$ is compact and hence there exists $z^{*} \in Z$ such that $d(x, Z)=d\left(x, z^{*}\right)$. Since $x \notin Z, d\left(x, z^{*}\right)>0$. However, there exists $\widetilde{z} \in Z$ such that $d(x, \widetilde{z})<d\left(x, z^{*}\right)$, a contradiction which proves the claim.

The connectedness of closed balls immediately implies the connectedness of open balls and of the whole space. Then $(X, d)$ is connected, locally connected and locally compact and then $(X, \tau)$ is a generalized Peano continuum.

Metric spaces whose metric is complete convex and satisfies (1), as in condition $\mathbf{b}$ of Theorem 1, have many further properties. In particular, as it can be immediately proved, these spaces are separable, second countable, $\sigma$-compact and arc-wise connected and, as Mazurkiewicz proved in [10], they are continuous image of the half-line $[0, \infty)$. Moreover, they are geodesic spaces, that is, there exists a geodesic path between any pair of distinct points (see [14], Section 2.6).

\section{On utility functions}

Let $\succeq$ be a continuous preference relation on a metric space $(X, d)$ and set, for every $x \in X$,

$$
C^{-}(x)=\{y \in X: x \succeq y\}, \quad O^{-}(x)=\{y \in X: x \succ y\} .
$$

Clearly $C^{-}(x)$ is closed and $O^{-}(x)$ is open. For every $x \in X$, we have

$$
\overline{O^{+}(x)} \subseteq C^{+}(x) \text { and } \overline{O^{-}(x)} \subseteq C^{-}(x)
$$

but, in general, equalities do not hold. 
We say that $\succeq$ is non-satiated on $X$ if, for every $x \in X$, there exists $x^{*} \in X$ such that $x^{*} \succ x$, otherwise we say $\succeq$ is satiated on $X$. Moreover we say that $\succeq$ is locally non-satiated (resp. locally non-hungry) at $x \in X$ if, for every $\varepsilon>0$, there exists $x^{*} \in B(x, \varepsilon)$ such that $x^{*} \succ x$ (resp. $\left.x \succ x^{*}\right)$.

Theorems 2 and 3 below describe how to build an upper semi-continuous utility function when the continuous preference relation is non-satiated and satiated respectively. The proof of Theorem 3 is omitted as it is a simple modification of the original proof by Arrow and Hahn (see [2], pp. 83-84).

Theorem 2. Let $(X, d)$ be a metric space satisfying (1) and (2) and let $\succeq$ be a non satiated continuous preference relation on $X$. Then, for every $\xi \in X$, the function $u_{\xi}: X \rightarrow \mathbb{R}$ defined as

$$
u_{\xi}(x)=\left\{\begin{aligned}
d\left(\xi, O^{+}(x)\right) & \text { if } \quad x \in C^{+}(\xi), \\
-d\left(\xi, C^{-}(x)\right) & \text { if } \quad x \in O^{-}(\xi),
\end{aligned}\right.
$$

represents $\succeq$ and is upper semi-continuous. Moreover, if

$$
\begin{gathered}
\text { for every } x \in C^{+}(\xi), \succeq \text { is locally non-satiated at } x, \\
\text { for every } x \in O^{-}(\xi) \cup\{\xi\}, \succeq \text { is locally non-hungry at } x,
\end{gathered}
$$

then $u_{\xi}$ is continuous.

Proof. First of all, note that the function $u_{\xi}$ is well defined. In fact, for every $x \in X$, the set $O^{+}(x)$ is nonempty as $\succeq$ is non-satiated and $C^{-}(x)$ is nonempty as it contains $x$. Moreover (1) implies that, for every $x \in X$ and for every nonempty closed set $C \subseteq X$, there exists $y_{C} \in C$ such that $d\left(x, y_{C}\right)=d(x, C)$ and hence, for every $x \in O^{-}(\xi), u_{\xi}(x)<0$. Of course, for every $x \in C^{+}(\xi), u_{\xi}(x) \geq 0$.

In order to prove that $u_{\xi}$ represents $\succeq$, let $x_{1}, x_{2} \in X$. Assume first that $x_{1} \sim x_{2}$. If $x_{1}, x_{2} \in C^{+}(\xi)$ then, as $O^{+}\left(x_{1}\right)=O^{+}\left(x_{2}\right)$, it follows $u_{\xi}\left(x_{1}\right)=u_{\xi}\left(x_{2}\right)$. If instead $x_{1}, x_{2} \in$ $O^{-}(\xi)$ then, as $C^{-}\left(x_{1}\right)=C^{-}\left(x_{2}\right)$, it follows $u_{\xi}\left(x_{1}\right)=u_{\xi}\left(x_{2}\right)$.

Assume now that $x_{1} \succ x_{2}$. If $x_{1}, x_{2} \in O^{-}(\xi)$, then there exists $y_{2} \in C^{-}\left(x_{2}\right)$ such that $u_{\xi}\left(x_{2}\right)=-d\left(\xi, C^{-}\left(x_{2}\right)\right)=-d\left(\xi, y_{2}\right)$. Hence $x_{1} \succ x_{2} \succeq y_{2}$ so that $y_{2} \in O^{-}\left(x_{1}\right)$. By (2) there exists $y_{1} \in O^{-}\left(x_{1}\right)$ such that $d\left(y_{1}, \xi\right)<d\left(y_{2}, \xi\right)$. As $O^{-}\left(x_{1}\right) \subseteq C^{-}\left(x_{1}\right)$ we have $d\left(\xi, O^{-}\left(x_{1}\right)\right) \geq$ $d\left(\xi, C^{-}\left(x_{1}\right)\right)$, hence

$$
u_{\xi}\left(x_{2}\right)=-d\left(\xi, y_{2}\right)<-d\left(\xi, y_{1}\right) \leq-d\left(\xi, O^{-}\left(x_{1}\right)\right) \leq-d\left(\xi, C^{-}\left(x_{1}\right)\right)=u_{\xi}\left(x_{1}\right) .
$$

If $x_{1}, x_{2} \in C^{+}(\xi)$, then there exists $y_{1} \in \overline{O^{+}\left(x_{1}\right)} \subseteq C^{+}\left(x_{1}\right)$ such that $u_{\xi}\left(x_{1}\right)=d\left(\xi, O^{+}\left(x_{1}\right)\right)=$ $d\left(\xi, y_{1}\right)$. Then $y_{1} \succeq x_{1} \succ x_{2}$ and hence $y_{1} \in O^{+}\left(x_{2}\right)$. By (2) there exists $y_{2} \in O^{+}\left(x_{2}\right)$ such that $d\left(y_{2}, \xi\right)<d\left(y_{1}, \xi\right)$. Hence

$$
u_{\xi}\left(x_{1}\right)=d\left(\xi, y_{1}\right)>d\left(\xi, y_{2}\right) \geq d\left(\xi, O^{+}\left(x_{2}\right)\right)=u_{\xi}\left(x_{2}\right) .
$$

Finally if $x_{1} \in C^{+}(\xi)$ and $x_{2} \in O^{-}(\xi)$, we have $u_{\xi}\left(x_{1}\right) \geq 0$ and $u_{\xi}\left(x_{2}\right)<0$, thus $u_{\xi}\left(x_{1}\right)>$ $u_{\xi}\left(x_{2}\right)$ and the proof that $u_{\xi}$ represents $\succeq$ is complete.

In order to prove $u_{\xi}$ is upper semi-continuous, let $x \in X$ and let $\left\{x_{n}\right\}_{n=1}^{\infty} \subseteq X$ be a sequence which converges to $x$ and such that the limit of $u_{\xi}\left(x_{n}\right)$ as $n$ goes to infinity exists (finite or infinite). We have to prove that

$$
u_{\xi}(x) \geq \lim _{n \rightarrow \infty} u_{\xi}\left(x_{n}\right) .
$$


Suppose first $x \in O^{-}(\xi)$. Without loss of generality we can assume that, for every $n \in \mathbb{N}$, $x_{n} \in O^{-}(\xi)$. For every $n \in \mathbb{N}$, there exists $y_{n} \in C^{-}\left(x_{n}\right)$ such that $u_{\xi}\left(x_{n}\right)=-d\left(\xi, C^{-}\left(x_{n}\right)\right)=$ $-d\left(\xi, y_{n}\right)$. Since $x_{n} \in C^{-}\left(x_{n}\right)$ implies $d\left(\xi, y_{n}\right) \leq d\left(\xi, x_{n}\right)$ and since $\left\{x_{n}\right\}_{n=1}^{\infty}$ converges, we have that the sequence $\left\{d\left(\xi, y_{n}\right)\right\}_{n=1}^{\infty}$ is bounded. By (1), the sequence $\left\{y_{n}\right\}_{n=1}^{\infty}$ admits a subsequence $\left\{y_{n_{k}}\right\}_{k=1}^{\infty}$ which converges to, say, $y$. We know that, for every $k \in \mathbb{N}, y_{n_{k}} \in$ $C^{-}\left(x_{n_{k}}\right), y_{n_{k}} \rightarrow y$ and $x_{n_{k}} \rightarrow x$. Then, the continuity of $\succeq$ implies $y \in C^{-}(x)$ and (5) follows since

$$
u_{\xi}(x)=-d\left(\xi, C^{-}(x)\right) \geq-d(\xi, y)=\lim _{k \rightarrow \infty}-d\left(\xi, y_{n_{k}}\right)=\lim _{k \rightarrow \infty} u_{\xi}\left(x_{n_{k}}\right)=\lim _{n \rightarrow \infty} u_{\xi}\left(x_{n}\right) .
$$

Suppose now $x \in C^{+}(\xi)$. If for infinitely many $n \in \mathbb{N}, x_{n} \in O^{-}(\xi)$ then (5) follows immediately. Without loss of generality, we can assume that $x_{n} \in C^{+}(\xi)$ for every $n \in \mathbb{N}$. We have $u_{\xi}(x)=d(\xi, y)$ for some $y \in \overline{O^{+}(x)}$ and, for every $\varepsilon>0$, there exists $y^{*} \in O^{+}(x)$ such that $d\left(y, y^{*}\right)<\varepsilon$. Clearly $y^{*} \succ x$ implies that, for every $n$ large enough, $y^{*} \succ x_{n}$ and

$$
u_{\xi}(x)=d(\xi, y) \geq d\left(\xi, y^{*}\right)-\varepsilon \geq d\left(\xi, O^{+}\left(x_{n}\right)\right)-\varepsilon=u_{\xi}\left(x_{n}\right)-\varepsilon .
$$

The above relation implies (5).

Assume now that (3) and (4) hold. In order to prove that $u_{\xi}$ is lower semi-continuous, let $x \in X$ and let $\left\{x_{n}\right\}_{n=1}^{\infty} \subseteq X$ be a sequence which converges to $x$ and such that the limit of $u_{\xi}\left(x_{n}\right)$ as $n$ goes to infinity exists (finite or infinite). We have to prove that

$$
u_{\xi}(x) \leq \lim _{n \rightarrow \infty} u_{\xi}\left(x_{n}\right)
$$

Suppose first that $x \in O^{-}(\xi)$. Without loss of generality we can assume $x_{n} \in O^{-}(\xi)$ for every $n \in \mathbb{N}$. We have $u_{\xi}(x)=-d(\xi, y)$ for some $y \in C^{-}(x)$, and, from (4), for every $\varepsilon>0$, there exists $y^{*} \in X$ such that $y \succ y^{*}$ and $d\left(y, y^{*}\right)<\varepsilon$. Clearly $x \succ y^{*}$ and then $x_{n} \succ y^{*}$ for every $n$ large enough. It follows

$$
\lim _{n \rightarrow \infty} u_{\xi}\left(x_{n}\right) \geq u_{\xi}\left(y^{*}\right)=-d\left(\xi, C^{-}\left(y^{*}\right)\right) \geq-d\left(\xi, y^{*}\right) \geq-d(\xi, y)-\varepsilon=u_{\xi}(x)-\varepsilon .
$$

As $\varepsilon$ is arbitrary (6) holds.

Suppose now $x \in O^{+}(\xi)$. Without loss of generality we can assume, for every $n \in \mathbb{N}, x_{n} \in$ $O^{+}(\xi)$. Note that (3) implies that, for every $y \in C^{+}(\xi), \overline{O^{+}(y)}=C^{+}(y)$ and hence $u_{\xi}(x)=$ $d\left(\xi, C^{+}(x)\right)$. For every $n \in \mathbb{N}$, there exists $y_{n} \in C^{+}\left(x_{n}\right)$ such that $u_{\xi}\left(x_{n}\right)=d\left(\xi, C^{+}\left(x_{n}\right)\right)=$ $d\left(\xi, y_{n}\right)$. Since $x_{n} \in C^{+}\left(x_{n}\right)$ implies $d\left(\xi, y_{n}\right) \leq d\left(\xi, x_{n}\right)$ and since $\left\{x_{n}\right\}_{n=1}^{\infty}$ converges, we have that the sequence $\left\{d\left(\xi, y_{n}\right)\right\}_{n=1}^{\infty}$ is bounded. By (1) the sequence $\left\{y_{n}\right\}_{n=1}^{\infty}$ admits a subsequence $\left\{y_{n_{k}}\right\}_{k=1}^{\infty}$ which converges to, say $y$. We know that, for every $k \in \mathbb{N}, y_{n_{k}} \in C^{+}\left(x_{n_{k}}\right), y_{n_{k}} \rightarrow y$ and $x_{n_{k}} \rightarrow x$. Then the continuity of $\succeq$ implies $y \in C^{+}(x)$ and then (6) follows since

$$
u_{\xi}(x)=d\left(\xi, C^{+}(x)\right) \leq d(\xi, y)=\lim _{k \rightarrow \infty} d\left(\xi, y_{n_{k}}\right)=\lim _{k \rightarrow \infty} u_{\xi}\left(x_{n_{k}}\right)=\lim _{n \rightarrow \infty} u_{\xi}\left(x_{n}\right) .
$$

Suppose finally $x \in C^{+}(\xi) \cap C^{-}(\xi)$, that is, $x \sim \xi$. Note that (3) implies $u_{\xi}(x)=0$. If for infinitely many $n \in \mathbb{N}, x_{n} \succeq x$ then (6) immediately follows. Consequently, without loss of generality, we can assume that, for every $n \in \mathbb{N}, x \succ x_{n}$, that is, $x_{n} \in O^{-}(\xi)$. Then, for every $n \in \mathbb{N}$, there exists $y_{n} \in C^{-}\left(x_{n}\right)$ such that $u_{\xi}\left(x_{n}\right)=-d\left(\xi, C^{-}\left(x_{n}\right)\right)=d\left(\xi, y_{n}\right)$. From 
(4), for every $\varepsilon>0$, there exists $y \in X$ such that $\xi \succ y$ and $d(\xi, y)<\varepsilon$. Since $O^{+}(y)$ is a neighborhood of $x$, if $n$ is large enough, then $x_{n} \succ y$ and

$$
\lim _{n \rightarrow \infty} u_{\xi}\left(x_{n}\right) \geq u_{\xi}(y)=-d\left(\xi, C^{-}(y)\right) \geq-d(\xi, y) \geq-\varepsilon .
$$

Since $u_{\xi}(x)=0$ and $\varepsilon$ is arbitrary, (6) holds.

Theorem 3. Let $(X, d)$ be a metric space satisfying (1) and (2) and $\succeq$ be a satiated continuous preference relation on $X$. Then, for every $\xi \in X$ such that, for every $y \in X, \xi \succeq y$, the function $u_{\xi}: X \rightarrow \mathbb{R}$ defined, for every $x \in X$, as $u_{\xi}(x)=-d\left(\xi, C^{-}(x)\right)$ represents $\succeq$ and is upper semi-continuous. Moreover if $\succeq$ is locally non-hungry at every point of $X$ then $u_{\xi}$ is continuous.

\section{References}

[1] J.C.R. Alcantud - G.B. Mehta, Constructive utility functions on Banach spaces, J. Math. Anal. Appl., 350 (2009), 590-600.

[2] K. Arrow - F. Hahn, General Competitive Analysis, Holden-Day, San Francisco, 1971.

[3] A.F. Beardon, Utility representation of continuous preferences, Econom. Theory, 10 (1997), 369-372.

[4] R. H. Bing, A convex metric for a locally connected continuum, Bull. Amer. Math. Soc., 55 (1949), 812-819.

[5] D.S. Bridges - G.B. Mehta, Representations of preference orderings, Lecture Notes in Economics and Mathematical Systems, 422, Springer, 1995.

[6] J.C. Candeal - E. Induráin - G.B. Mehta, Utility functions on locally connected spaces, J. Math. Econom., 40 (2004), 701-711.

[7] G. Debreu, Continuity properties of paretian utility, Internat. Econom. Rev., 5 (1964), 285-293.

[8] S. Eilenberg, Ordered topological spaces, Amer. J. Math., LXIII (1941), 39-45.

[9] A. Lelek - J. Mycielski, On convex metric spaces IV, Fund. Math., 61, (1967), 171176.

[10] S. Mazurkiewicz, Sur les lignes de Jordan, Fund. Math., 1 (1920), 166-209.

[11] K. Menger, Untersuchungen über allgemeine metrik, Math. Ann., 100 (1928), 75-163.

[12] E.E. MoIse, Grille decomposition and convexification theorems for compact metric locally connected continua, Bull. Amer. Math Soc., 55 (1949), 1111-1121.

[13] P.K. Monteiro, Some results on the existence of utility functions on path connected spaces, J. Math. Econom., 16 (1987), 147-156. 
[14] A. Papadopoulos, Metric spaces, convexity and nonpositive curvature, IRMA Lectures in Mathematics and Theoretical Physics, 6. European Mathematical Society (EMS), Zürich, 2005.

[15] A. Tominaga - T. Tanaka, Convexification of locally connected generalized continua, J. Sci. Hiroshima Univ. Ser. A, 19 (1955), 301-306. 